\title{
Contexto y actividad física por género del profesorado de educación física
}

\section{Context and physical activity by gender of physical education teachers}

\author{
HALL-LÓPEZ, Javier Arturo ${ }^{1}$ \\ OCHOA-MARTÍNEZ, Paulina Yesica²
}

\begin{abstract}
Resumen
El estudio evaluó el contexto y actividad física en 82 clases de educación física en primaria mediante el sistema para observar el tiempo de instrucción de actividad física (SOFIT) por sus siglas en inglés, comparando la conducción de las clases por el profesorado de acuerdo con el género. El análisis estadístico realizado con la prueba t Student para muestras independientes mostró que en el contexto docente las profesoras se inclinan en sus estrategias hacia el juego como recurso didáctico y los profesores tienden al acondicionamiento físico, con mayor práctica de actividad física moderada en clases dirigidas por profesoras y mayor intensidad vigorosa en las de los profesores.

Palabras clave: género; educación física; primaria; profesorado.
\end{abstract}

\begin{abstract}
The study evaluated the context and physical activity in 82 physical education classes in primary school using the system for observing physical activity instruction time (SOFIT), comparing the conduct of classes by teachers according to gender, the statistical analysis carried out with the Student test for independent samples showed that in the teaching context the teachers lean in their strategies towards the game as a didactic resource and the teachers tend towards physical conditioning, with more moderate physical activity practiced in directed classes by female teachers and more vigorous intensity by teachers.

Key words: gender; physical education; elementary scholl; teachers.
\end{abstract}

\section{Introducción}

El Banco Mundial, en su informe 2018 sobre el desarrollo, promueve la importancia de aprendizajes tendientes a la igualdad de género. También la Organización de las Naciones Unidas (ONU, 2015), en sus objetivos para el desarrollo sostenible establece a la educación de calidad e igualdad de género. En el ámbito de la educación física, la Organización de las Naciones Unidas para la Educación, la Ciencia y la Cultura (UNESCO), exhorta en su guía para responsables políticos que laboran en torno la educación física establecer acciones de igualdad de género para el logro en la calidad educativa (Mclennan, y Thompson, 2015), dichas acciones deben realizarse en diversas etapas educativas con contenidos e información para las estudiantes que mejore su confianza para

\footnotetext{
${ }^{1}$ Doctor en Investigación en la Enseñanza y el Aprendizaje de la Ciencias Experimentales, Sociales, Matemáticas y de la Actividad Física y Deportiva por la Universidad de Huelva; Doctor en Medicina del Deporte por la Universidad Católica Nuestra Señora de la Asunción. Profesor-Investigador de Tiempo Completo de la Facultad de Deportes, Universidad Autónoma de Baja California. javierhall@uabc.edu.mx

2 Doctora en Investigación en la Enseñanza y el Aprendizaje de la Ciencias Experimentales, Sociales, Matemáticas y de la Actividad Física y Deportiva por la Universidad de Huelva, España; Doctora en Medicina del Deporte por la Universidad Católica Nuestra Señora de la Asunción. Profesora-Investigadora de Tiempo Completo de la Facultad de Deportes, Universidad Autónoma de Baja California. pochoa@uabc.edu.mx
} 
practicar ejercicio, actividad física y deporte (Frizzo, y Da Silva Souza, 2019). En el entorno del ejercicio físico se ha comprobado que las adolescentes de educación secundaria, bachillerato y universidad practican en menor medida que los hombres de su misma edad (Goncalves, y Martínez, 2018) así como tienen menores hábitos de práctica deportiva (Calvo-Ortega, y Perrino-Peña, 2017). Por lo que es importante acciones educativas con proyectos pedagógicos que disminuyan esas tendencias desde etapas educativas previas como la primaria (Dornelles, \& Wenetz, 2019). Respecto a la asignatura de educación física, estudios han valorado una menor intención por las estudiantes para participar activamente en tareas motrices (Gil Quintana, y Etxebeste Otegi, 2019), así como realizar menor actividad física en clase de educación física (Fröberg, et al. 2017), de manera global al analizar la jornada escolar investigaciones han observado el mismo patrón reportando mayores conductas sedentarias en el estudiantado de género femenino (Da Costa, et al. 2017; Tanaka, et al. 2018), esto también incluyendo el tiempo que se dedica al recreo (Viciana, et al. 2016).

Investigaciones de tipo cualitativo, han analizado al profesorado de educación física identificando cierta masculinidad y femineidad como productos de relaciones de poder marcadamente desiguales y asimétricas (Scharagrodsky, 2004) otra investigación con enfoque similar analizó al profesorado de educación física por género, e identificó en la comunicación y expresión con el estudiantado un polaridad que tendía a la desigualdad de género (Mariano, y Altmann, 2016). Otro estudio establece la misma tendencia reportando que el profesorado de educación física percibe que los contenidos son menos apropiados para la participación de las mujeres en comparación a los hombres (Castejón, y Giménez, 2015). Por lo anterior es importante dentro de las implicaciones del trabajo docente, establecer criterios epistemológicos que orienten hacia la solución de problemáticas actuales desde la educación física (Vargas, y Moreira, 2012). Derivando de esto la importancia de la formación permanente del profesorado en el ámbito de la diversidad e igualdad de género (Rodríguez, et al. 2019), ya se ha referido la importancia e influencia ejercida por el profesor, para la conducción contenidos que evitan estereotipos relacionados al género en educación física (Solmon, 2014).

La Encuesta Nacional de Salud y Nutrición MC2016 reporta que el 17.2\% de los habitantes de México entre 1014 años de edad se clasifican como activos acorde a las recomendaciones de la Organización Mundial para la Salud, y estos valores son menores en mujeres con un $12.7 \%$ comparándose con hombres con un 21.8\% (Medina, et al. 2017); de igual forma en materia de cultura física se ha identificado un desbalance de género, observando en las mujeres mayores barreras para realizar actividad física en sus diferentes modalidades (Flores Fernández, 2019).

En México, se han realizado estudios que han evaluado el contexto y la actividad física en las clases de educación física en educación primaria, utilizando como instrumento el System for Observing fitness and Instruction Time (SOFIT) (Mckenzie, Sallis, \& Nader, 1992; Mckenzie, 2002), Sistema para observar el tiempo de instrucción de actividad física por sus siglas en inglés, reportando como resultado una tendencia mayor en el contexto educativo guiado por el profesorado hacia la administración y el juego (Hall-López, J Sáenz-Lopez Buñuel, y Almagro, 2020), la actividad física reportada con intensidad moderada a vigorosa se reporta con valores menores al $50 \%$ del tiempo de la clase, es decir el profesorado promueve menos de la mitad de la clase que los estudiantes permanezcan activos con un gasto energético similar a caminar o correr (Hall-López, et al. 2018). Estos estudios no han reportado las diferencias por género.

El sistema educativo mexicano y el programa actual de educación física de la Secretaría de Educación Pública (SEP, 2017), establecen en los perfiles de egreso la convivencia y ciudadanía. Podría promoverse, desde el ámbito de la educación física, aspectos como igualdad de género, pero es importante identificar el contexto y la actividad física promovido por el profesor o profesora de educación física en educación primaria, y contar con información para intervenciones educativas efectivas tendientes a minimizar la desigualdad establecida en el estado del arte. 


\section{Metodología}

La presente investigación se registró y aprobó por la Universidad Autónoma de Baja California en la Coordinación de Posgrado e Investigación, siguiendo los principios éticos de investigación en humanos establecidos por la declaración de Helsinki (Rupali, 2005); Mediante una metodología de tipo transversal descriptivo comparativo, con muestreo probabilístico por conveniencia (Thomas, et al. 2015). Para el logro del objetivo fueron evaluadas 82 clases de educación física de la zona urbana de la ciudad de Mexicali, Baja California. México. Valorando clases de educación física conducidas por el profesorado a de estudiantes de primero a sexto de primaria con una edad promedio de $8.7 \pm .7$ años, dichas clases fueron realizadas por 40 profesoras de educación física mujeres con una antigüedad promedio en el servicio profesional docente de $7.9 \pm 4.2$ años y 42 por profesores de educación física hombres con una antigüedad promedio en el servicio profesional docente de $12.1 \pm 9.5$ años.

\subsection{Instrumentos y procedimientos}

El contexto y la actividad física se evaluaron acorde a los procedimientos del Sistema para Observar el Tiempo de Instrucción de Actividad Física (SOFIT: System for Observing fitness and Instruction Time) (Mckenzie, et al. 1992; Mckenzie, 2002); El instrumento para evaluar al profesorado se valora de manera cuantitativa, por medio de cuatro estudiantes de la clase elegidos aleatoriamente, dos hombres y dos mujeres. De las ochenta y dos clases de educación física evaluadas, participaron 328 estudiantes, cada uno observado en secuencia rotatoria de doce intervalos durante veinte segundos, repitiendo las observaciones durante toda la clase, el seguimiento de la evaluación fue mediante un audio que establece el protocolo SOFIT, para el registro de la actividad, con un reproductor de audio, durante 50 minutos de la duración de la clase como lo establece la normatividad. En el contexto de la clase de educación física dirigida por el profesorado, se identificaron siete códigos M.- Contenido general, P.- Conocimiento específico, K.- Conocimiento general, F.- Acondicionamiento físico, S.- Desarrollo de habilidades, G.- Juego, O.- Otros aspectos de la clase.

De manera simultánea para evaluar la actividad física de acuerdo al método SOFIT se siguieron los códigos 1.Acostado, 2.- Sentado, 3.- Parado, 4.- Caminando, y 5.- Muy activo que corresponde a correr o una actividad con un gasto energético mayor. El índice de actividad física moderada a vigorosa realizado en la clase del profesor sumando porcentualmente los códigos 4.- Caminando y 5.- Muy activo del total del tiempo de la clase de educación física. De manera simultánea con SOFIT se evaluó la duración en minutos de la clase de educación física registrada mediante cronómetro.

La confiabilidad inter evaluación fue de un valor Kappa .86, de dos evaluadores con conocimiento en los procedimientos técnicos del instrumento y memorizaron las definiciones operativas de los códigos referentes al contexto y actividad física.

\subsection{Análisis de los datos}

La homogeneidad de la varianza de los datos y la normalidad en los grupos, se evaluó con el test de KolmogorovSmirnov estableciendo un grado de significancia de P-Valor $\geq 0.05$, se realizó estadística descriptiva y se determinó el porcentaje de diferencia $(\Delta \%)$ entre la clase de educación física impartida por las profesoras y profesores ([(Media-2 - Media-1)/Media-1] x 100) (Vincent, 2012). Para el cálculo la igualdad de la varianza, se utilizó la prueba t Student para muestras independientes estableciendo un nivel de $\alpha \leq 0.05$, utilizando el Paquete Estadístico para las Ciencias Sociales (SPSS), versión 23.0 para Windows (IBM Corporation, New York, USA); se establecieron como variables fijas acorde al sexo dos grupos 1) Profesoras o 2) Profesores y las variables del contexto y actividad física como variables aleatorias numéricas, en las clases de educación física en primaria. 


\section{Resultados}

La duración promedio de las ochenta y dos clases de educación física en educación primaria fue de $40.3 \pm 7.1$ minutos con un rango de 15 a 52. La distribución porcentual del contexto en las 82 clases de educación física en primaria por las profesoras y los profesores, se presenta en la Figura 1. La estadística descriptiva de la actividad física promedio, desviación estándar, y homogeneidad de la varianza son observados en la tabla 1.

Figura 1

Distribución porcentual del contexto de las 82 clases de educación física en primaria por las profesoras y los profesores.

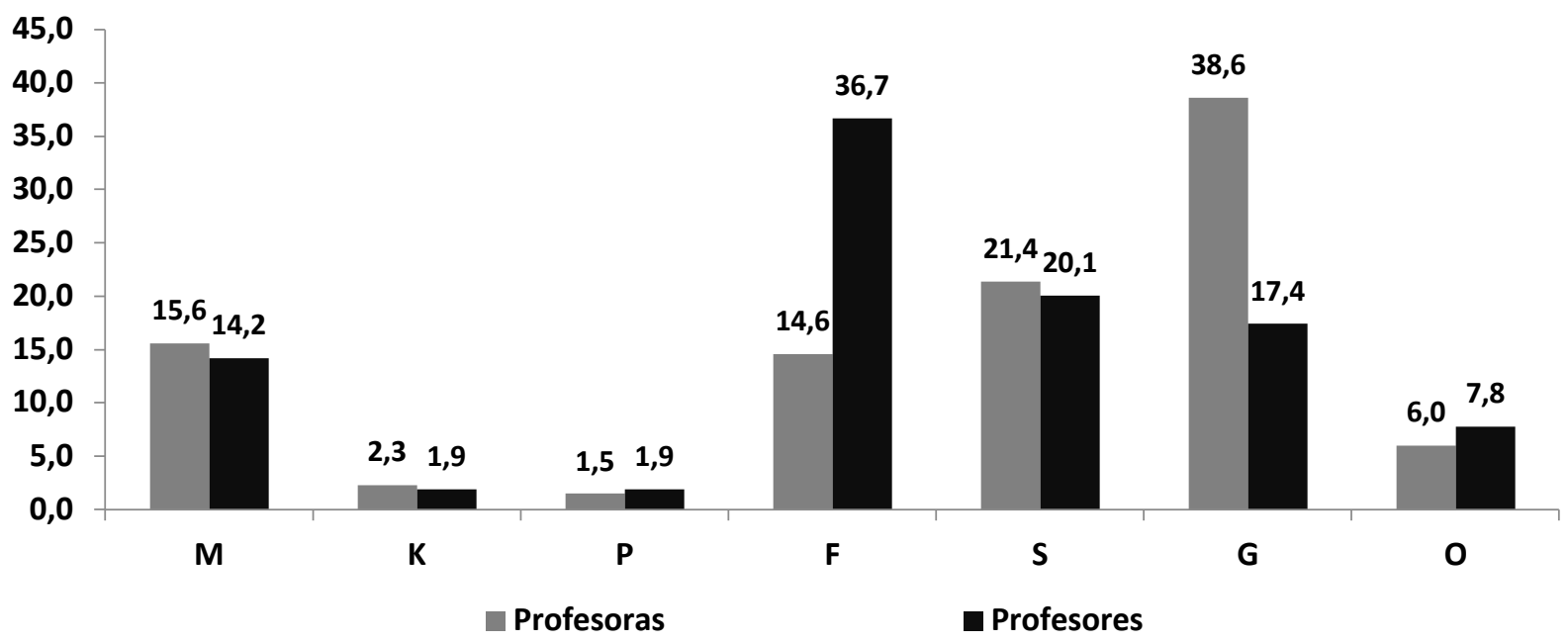

Nota: Distribución porcentual de la intensidad las clases de educación física impartidas por los profesores de educación física de acuerdo al género, utilizando como instrumento de evaluación: el sistema para observar el tiempo de instrucción de actividad física SOFIT; Clasificando a los códigos como M.- Contenido general, P.- Conocimiento específico, K.- Conocimiento general, F.- Acondicionamiento físico, S.- Desarrollo de habilidades, G.- Juego, O.- Otros. Utilizando como instrumento de evaluación: el sistema para observar el tiempo de instrucción de actividad física SOFIT (Mckenzie, 2002).

\section{Tabla 1}

Estadística descriptiva y homogeneidad de la varianza en de las profesoras profesores y los profesores de educación física en primaria $\mathrm{n}=82$.

\begin{tabular}{|c|c|c|c|c|}
\hline \multirow{3}{*}{$\begin{array}{c}\text { Variables } \\
\text { SOFIT }\end{array}$} & \multicolumn{4}{|c|}{ Clases de Educación Física en secundaria } \\
\hline & \multicolumn{2}{|r|}{ Profesoras $n=40$} & \multicolumn{2}{|c|}{ Profesores $n=42$} \\
\hline & $M \pm D E$ & Test Kolmogorov-Smirnov & $M \pm D E$ & Test Kolmogorov-Smirnov \\
\hline 1.- Acostado (\%) & $4.6 \pm .2$ & .361 & $4.1 \pm .3$ & .317 \\
\hline 2.-Sentado (\%) & $16.2 \pm 2.1$ & .527 & $13.6 \pm 4.1$ & .317 \\
\hline 3.- Parado (\%) & $37.1 \pm 6.2$ & .630 & $35.9 \pm 5.6$ & .852 \\
\hline 4.- Caminando (\%) & $35.3 \pm 5.6$ & .243 & $29.9 \pm 6.3$ & .427 \\
\hline 5.- Muy activo (\%) & $10.4 \pm 1.2$ & .713 & $16.5 \pm 1.1$ & .377 \\
\hline \multicolumn{5}{|c|}{$\begin{array}{l}\text { Nota: Tabla comparativa por género de la media, desviación estándar ( } \pm \text { ) normalidad y homogeneidac } \\
\text { de la varianza entre grupos mediante el test estadístico Test Kolmogorov-Smirnov, *p } \geq 0.05 \text {. Variables } \\
\text { Distribución porcentual de la intensidad las clases de educación física evaluada por SOFIT; Clasificando } \\
\text { los códigos como 1.- acostado, 2.- sentado, 3.- parado, 4.- caminando, y 5.- muy activo que corresponde } \\
\text { a correr o una actividad con un gasto energético mayor. Evaluada, mediante el sistema para observar e } \\
\text { tiempo de instrucción de actividad física SOFIT (Mckenzie, 2002). }\end{array}$} \\
\hline
\end{tabular}


A partir del método utilizado se estableció una prueba de hipótesis alterna y otra nula:

La hipótesis alterna: Existen diferencias significativas entre los valores del contexto y actividad física por género en el profesorado que imparte clases de educación física en primaria.

Hipótesis nula: No Existen diferencias significativas entre los valores del contexto y actividad física por género en el profesorado que imparte clases de educación física en primaria.

La valoración de la igualdad de la varianza entre grupos mediante el test t-Student, reportó diferencias significativas en el contexto de la clase de educación física resultando significativa juego código G, con valores mayores en las profesoras del género femenino (0.001) con 36.8 vs. 17.4 y el acondicionamiento físico código F, conducido por los profesores del género masculino (0.001) $36.7 \%$ vs. $14.6 \%$. La actividad física con intensidad moderada código 4, resultó con mayores promedios en el profesorado de género femenino (0.002), de igual forma la actividad física con intensidad vigorosa código 5, fue mayor en el profesorado de género masculino (0.005), al determinar el índice de actividad física moderada a vigorosa (suma de códigos 4 y 5) no existieron diferencias significativas entre grupos.

\subsection{Discusión}

El resultado principal en el estudio fue que al comparar las clases de educación física por género, las profesoras tienden en mayor medida a conducir acciones pedagógicas mediante juego (54.9 $\Delta \%$ ) que los profesores hombres, mientras que el profesorado de género masculino tiende a impartir acciones pedagógicas de acondicionamiento físico (59.9 $\Delta \%$ ). El índice de actividad física moderada a vigorosa sumando los códigos 4 y 5 no se encontraron diferencias significativas, aun sin embargo respecto a la intensidad moderada se observó que las profesoras fomentan mayor actividad física de intensidad moderada (18.1 $\Delta \%)$, mientras que el profesorado de género masculino fomenta mayores valores de actividad vigorosa (34.8 $\Delta \%$ ), se observa que el profesorado de género masculino fomenta la actividad física vigorosa (37 $\Delta \%$ ). Los valores encontrados se asocian a la investigación realizada por Beltrán Véliz, Barros Ketterer, \& Carter Thuillier, 2020, al reportar una educación centrada en la mejora de la condición física. De manera global índice de actividad física moderada a vigorosa generado en las clases de educación física impartidas por profesores fue $46 \%$, que al compararse con los estándares establecidos internacionalmente por el National Association for Sport and Physical Education (NASPE) no lograron una intensidad moderada a vigorosa por lo menos el 50\% del tiempo de la clase (Banville, 2006, Baghurst, et al. 2015). En México se han realizado investigaciones con similar abordaje metodológico con índices de actividad física similares a los resultados en el estudio (Hall-López, 2020; Hall-Lopez, et al. 2020).

Al realizar un análisis del estado de la cuestión, se ha demostrado la influencia del profesorado de educación física es importante en la conducción contenidos que evitan estereotipos asociados a la desigualdad de género (Scharagrodsky, 2004, Solmon, 2014), se han identificado proyectos escolares que promueven la diversidad e igualdad de género (Dornelles, y Wenetz, 2019; algunos de ellos enfocados en la promoción del profesor para la participación equitativa de actividad física entre el estudiantado, tomando en cuenta modelos de deportistas exitosas (Post, \& Palacios, 2019), otro estudio aborda la importancia del profesorado de educación física y los estudiantes de género masculino para lograr un incremento en la actividad física moderada vigorosa de las mujeres (Bronikowski, et al. 2015). Por lo anterior, la UNESCO se ha recomendado estrategias didácticas para minimizar la desigualdad de género. Y en el profesorado se ha tomado volar a la formación superior en todas las áreas del conocimiento para abordar contenidos que tiendan a cerrar esa brecha (Rodríguez, et al. 2019; Pastor Gosálbez, et al. 2020), incluyendo a la educación física que favorecen entornos adecuados de aprendizaje con una perspectiva de género (Payeras, et al. 2016); y brindando importancia en el contexto social a la mujer como profesional de la educación física (Schwengber, y Meyer, 2011; Ibacache, \& Iniguez-Rueda, 2019; Ramírez,. Manosalvas, y Cardenas, 2019). 
La Secretaría de Educación Pública en México, dentro de sus perfiles de egreso en los programas de educación básica, está la colaboración y el trabajo en equipo así como la atención al cuerpo y la salud, y dentro de sus componentes curriculares esta la educación física como área de desarrollo personal social (SEP, 2017), por lo cual pueden tomar en cuenta esos elementos pedagógicos para que el profesorado dirija clases de educación física de manera intencionada generando oportunidades de participar en movimiento con participación equitativa en los estudiantes de ambos géneros. Por lo anterior la investigación pretende brindar información importante al profesorado, en la toma decisiones, para la conducción de contenidos, aplicación de estrategias didácticas, y diseño de tareas motrices en la clase de educación física, tomando en cuenta la perspectiva de género que atiendan una alfabetización corporal (Bolanos-Motta, et al. 2018).

\section{Conclusiones}

El estudio identificó diversidad en el contexto educativo que dirige el profesorado de educación física en primaria acorde al género. Las profesoras se inclinan al juego como recurso didáctico y el contexto docente, mientras que los profesores tienden al acondicionamiento físico. También se encontró mayor presencia de actividad física moderada en las clases dirigidas por las profesoras y mayor intensidad vigorosa en las clases dirigidas por los profesores. En promedio no se logró ni el 50\% del tiempo de la clase de educación física en actividad física moderada a vigorosa.

\section{Referencias bibliográficas}

Banville, D. (2006). Analysis of exchanges between novice and cooperating teachers during internships using the NCATE/NASPE standards for teacher preparation in physical education as guidelines. Research Quarterly for Exercise and Sport, 77(2), 208-221. doi:10.1080/02701367.2006.10599355

Baghurst, T.; Langley, J.; y Bishop, J. (2015). Physical Educators' Perceptions of Their Use of NASPE Standards. The Physical Educator, 72(5), 324-341. doi:https://doi.org/10.18666/TPE-2015-V72-I5-6194

Beltrán Véliz, J. C.; Barros Ketterer, J. I.; y Carter Thuillier, B. I. (2020). Racionalidad técnica-instrumental en educación física. Un estudio cualitativo en contexto chileno. Revista Espacios, 41(04), 19-29. http://revistaespacios.com/a20v41n04/a20v41n04p19.pdf

Bolanos-Motta, J. I.; Perez-Rodriguez, M. A.; y Casallas-Forero, E. (2018). Alfabetización Corporal. Una propuesta de aula desde la psicomotricidad. Estudios pedagógicos, Valdivia, 44(3), 23-

34. https://doi.org/10.4067/S0718-07052018000300023

Bronikowski, M.;Bronikowska, M.; Laudanska-Krzeminska, I.; Kantanista, A.; Morina, B.; y Vehapi, S. (2015). PE Teacher and Classmate Support in Level of Physical Activity: The Role of Sex and BMI Status in Adolescents from Kosovo. BioMed Research International, 290349, 1-8. doi: 10.1155/2015/290349.

Calvo-Ortega, E.; y Perrino-Peña, M. (2017). Hábitos físico-deportivos dos adolescentes de Castilla e León. Movimento (ESEFID/UFRGS), Porto Alegre, 23(4), 1341-1352. https://doi.org/10.22456/19828918.71852

Castejón, F. J.; y Giménez, F. J. (2015). Teachers' perceptions of physical education content and influences on gender differences. Motriz, 21(4), 375-385. https://doi.org/10.1590/S1980-65742015000400006.

Da Costa, B.; Silva, S. K.; Altenburg De Assis, M. A.; y George, M. (2019). Sedentary behavior during school-time: Sociodemographic, weight status, physical education class, and school performance correlates in Brazilian schoolchildren. Journal of Science and Medicine in Sport, 20(1), 70-74. doi: 10.1016/j.jsams.2016.06.004.

Dornelles, P. G.; y Wenetz, I. (2019). Projeto gênero e diversidade na escola: a gendered approach on the project. Cadernos de Pesquisa, São Paulo, 49(173), 226-243. https://doi.org/10.1590/198053146074. 
Flores Fernández, Z. (2019). Mujer y deporte en México. Hacia una igualdad sustancial. Women and sport in México. Towards a substantive equality. Retos, 0(37), 222-226, https://recyt.fecyt.es/index.php/retos/article/view/71684.

Frizzo, G.; y Souza, M. D. S. (2019). Educação física nas diretrizes da unesco: o paradigma da aptidão física e da saúde na formação do capital humano. Movimento (ESEFID/UFRGS), Porto Alegre, 25(10), 1-12. doi: https://doi.org/10.22456/1982-8918.76037

Fröberg, A.; Raustorp, A.; Pagels, P.; y Larsson, C. (2017). Levels of physical activity during physical education lessons in Sweden. Acta Paediatrica, 106(1), 135-141. https://doi.org/10.1111/apa.13551

Gil Quintana, J.; y Etxebeste Otegi, J. (2019). Igualdade de gênero e análise da comunicação motora nas tarefas de educação física. Movimento (ESEFID/UFRGS), Porto Alegre, 25(10), 1-13. doi: https://doi.org/10.22456/1982-8918.85297

Goncalves, V. O.; y Martinez, J. P. (2018). Género y práctica de ejercicio físico de adolescentes y universitarios. Cadernos de Pesquisa, São Paulo, 48(170), 11141128, https://doi.org/10.1590/198053145588.

Hall-López, J. A. (2020). Rural-Urban differences in perception of effort and physical activity of adolescents in physical education. Revista Espacios, 41(26), 349-356. http://ww.revistaespacios.com/a20v41n26/a20v41n26p29.pdf

Hall-Lopez, J. A.; Ochoa-Martinez, P. Y.; López Campos, C. E.; y Alarcon-Meza, E. I. (2020). Perceived exertion and moderate to vigorous physical activity in middle school students according to the physical education teachers' experience. Facta Universitatis, Series Physical Education and Sport, 18(1), 179-188. https://doi.org/10.22190/FUPES2002220150

Hall-López, J. A., Sáenz-Lopez Buñuel, P. \& Almagro, B. (2020). Actividad Física Moderada a Vigorosa en Educación Física. Kronos Revista Científica de Actividad Física y Deporte, 19(1), 1-2. https://revistakronos.info/articulo/actividad-fisica-moderada-a-vigorosa-en-educacion-fisica-2777-saq5ef3f1a1924bd

Hall-López, J. A., Ochoa-Martínez, P. Y., Macías Castro, R., Zuñiga Burruel, R., \& Sáenz-López Buñuel, P. (2018). Actividad física moderada a vigorosa en educación física y recreo en estudiantes de primaria y secundaria de la frontera México-USA. Sportis: Revista Técnico-Científica del Deporte Escolar, Educación Física y Psicomotricidad, 4(3), 426-442. https://doi.org/10.17979/sportis.2018.4.3.3175

Ibacache, J.; y Espinoza Iniguez-Rueda, L. (2019). Mujeres profesionales del sexo: prácticas reflexivas y posiciones en el campo. Cadernos Pagu, Campinas, 56, 1-31. https://doi.org/10.1590/18094449201900560013.

Mariano, M.; y Altmann, H. (2016). Educação Física na Educação Infantil: educando crianças ou meninos e meninas?. Cadernos Pagu, Campinas, 46, 411-438. https://doi.org/10.1590/18094449201600460411.

Mckenzie, T. (2002). SOFIT. System for Observing Fitness Instruction Time. Overview and Training Manual. San Diego, CA: San Diego State University, https://thomckenzie.com/useful-tools/observation/

Mckenzie, T.; Sallis, J.; y Nader, P. (1992). Sofit-System for Observing Fitness Instruction Time. Journal of Teaching in Physical Education, 11(2), 195-205. https://doi.org/10.1123/jtpe.11.2.195

Mclennan, N.; y Thompson, J. (2015). Promoting Quality Physical Education Policy. Putting the Quality in Physical Education. United Nations Educational, Scientific and Cultural Organization. UNESCO.

http://www.unesco.org/new/es/social-and-human-sciences/themes/physical-education-and-sport/policyproject/ 
Medina, C.; Jáuregui, A.; Campos-Nonato, I.; y Barquera, S. (2018). Prevalencia y tendencias de actividad física en niños y adolescentes: resultados de Ensanut 2012 y Ensanut MC 2016. Salud Pública de México, 60(3), 263-271. http://saludpublica.mx/index.php/spm/article/view/8819.

Organización de las Naciones Unidas (ONU). La UNESCO y los Objetivos de Desarrollo Sostenible, Educación e igualdad de género, 2015. https://es.unesco.org/themes/educacion-igualdad-genero

Pastor Gosálbez, I.; Acosta Sarmiento, A.; Torres Coronas, T.; y Calvo Merino, M. (2020). Los planes de igualdad en las universidades españolas. Situación actual y retos de futuro. Educación XX1, 23(1), 147-172, doi:https://doi.org/10.5944/educxx1.23873.

Payeras, P. S.; Vizcarra Morales, M. T.; Garay Ibáñez De Elejalde, B.; Prat Grau, M.; y Soler Prat, S. (2016). Análise do discurso de gênero nas matrizes curriculares sobre as ciências da atividade física e do desporto. Movimento (ESEFID/UFRGS), Porto Alegre, 22(3), 821-834. https://doi.org/10.22456/19828918.56959

Post, P.; y Palacios, R. (2019). Aggie Play: A Gender-Relevant Physical Activity Program for Girls. Journal of Sport and Exercise Psychology, 41(4), 194-205. doi: 10.1123/jsep.2018-0299.

Ramírez, R. F.; Manosalvas, M. I.; y Cardenas, O. S. (2019). Estereotipos de género y su impacto en la educación de la mujer en Latinoamérica y el Ecuador. Revista Espacios, 40(41) 29-35. http://www.revistaespacios.com/a19v40n41/a19v40n41p29.pdf

Rodríguez, R.; Serrano Gracia, E.; Pérez Chan, M.; y Camargo, A. C. (2019). Respeto e igualdad de género en la formación inicial docente. Cadernos de Pesquisa, São Paulo, 49(174), 152166. https://doi.org/10.1590/198053146162.

Rupali, G. (2005). Research involving children: regulations, review boards and reform. Journal of Health Care Law and Policy, 8(2), 264-330. https://digitalcommons.law.umaryland.edu/jhclp/vol8/iss2/6

Secretaría de Educación Pública (SEP) Aprendizajes Clave para la Educación Integral. Educación Física. Educación Básica. Plan y Programas de Estudio y sugerencias de evaluación. Primera edición, Ciudad de México, 2017. https://www.gob.mx/sep/articulos/aprendizajes-clave-para-la-educacion-integral?idiom=es

Scharagrodsky, P. A. ( 2004). Juntos pero no revueltos: la educación física mixta en clave de género. Cadernos de Pesquisa, São Paulo, 34(121), 59-76. https://doi.org/10.1590/S0100-15742004000100004.

Schwengber, M. S.; Vione Y Meyer, D. E. (2011). Discursos que conformam corpos grávidos: da medicina à educação física. Cadernos Pagu, Campinas, 36, 283-314. https://doi.org/10.1590/S010483332011000100011.

Solmon, M. (2015). Physical education, sports, and gender in schools. Advances in child development and behavior, 10, 117-150.https://doi.org/10.1016/bs.acdb.2014.04.006

Tanaka, C.; Tanaka, M.; Y Tanaka, S. (2018). Objectively evaluated physical activity and sedentary time in primary school children by gender, grade and types of physical education lessons. BMC Public Health, 18( 1), 1-10.: 10.1186/s12889-018-5910-y.

The World Bank Group (WBG). The World Development Report 2018 (WDR 2018) LEARNING to Realize Education's Promise is the first ever devoted entirely to education 2018. https://www.worldbank.org/en/publication/wdr2018

Thomas, J.; Nelson, J.; y Silverman, S. (2015). Research Methods in Physical Activity (7th. Ed.). Human Kinetics. Champaign, Ilinois: Human Kinetics. 
Vargas, C. P.; y Moreira, A. F. B. (2012). A crise epistemológica na educação física: implicações no trabalho docente. Cadernos de Pesquisa, São Paulo, 42(146), 408-427. https://doi.org/10.1590/S010015742012000200006.

Viciana, J.; Mayorga-Vega, D.; y Martínez-Baena A. Moderate-to-Vigorous Physical Activity Levels in Physical Education, School Recess, and After-School Time: Influence of Gender, Age, and Weight Status. Journal of Physical Activity \& Health, 13(10), 1117-1123. doi:https://doi.org/10.1123/jpah.2015-0537

Vincent, W. (2012). Statistics in kinesiology (4nd Ed.). Champaign, Ilinois: Human Kinetics.

Esta obra está bajo una Licencia Creative Commons
Attribución-NoCommercial 4.0 International
\[ (\text { cc) B EY-NC } \]

